\title{
Comparative Analysis of Anthropometric Methods: Past, Present, and Future
}

\author{
Alfredo BALLESTER ${ }^{1}$, Warren WRIGHT ${ }^{2}$, Jorge VALERO ${ }^{1}$, Alice BULLAS ${ }^{3}$, \\ Tim DEVLIN ${ }^{4}$, Emma SCOTT ${ }^{5}$, Carol MCDONALD ${ }^{6}$ \\ ${ }^{1}$ Instituto de Biomecảnica, Universitat Politècnica de València, València, Spain; \\ 2 Size Stream LLC, Cary, NC, USA; \\ ${ }^{3}$ Centre for Sports Engineering Research, Sheffield Hallam University, UK; \\ ${ }^{4}$ Yellowjacket Technologies, Berlin, Germany; \\ ${ }^{5}$ Fashion Should Empower, Vancouver Island, BC, Canada; \\ ${ }^{6}$ Gneiss Concept, Washougal, WA,USA \\ https://doi.org/10.15221/20.28
}

Keywords: 3D body scanning, manual measurement, surface imaging, optical imaging

\begin{abstract}
3D imaging has experienced rapid market growth since its development in the 1980's. There are now many types of 3D surface imaging systems - each using different hardware, software, computer vision techniques and landmarks resulting in differing measurement locations and degrees of accuracy and precision.
\end{abstract}

Whilst manufacturers and independent studies have evaluated systemic and random errors of particular 3D surface imaging systems, diverse factors such as population samples, landmarking, measurement definitions, data cleaning processes or analysis methods inhibit informed judgements between systems and against manually collected data.

The IEEE 3D Body Processing Industry Connections (3DBP IC) Group is developing recommendations for 3D body processing interoperability to enable an ecosystem from consumers to creators to use and interchange 3D human body anthropometric information. The Quality subgroup is focused on developing preliminary methods, tools, benchmarks, resources, and testing procedures to define and quantify the quality of $3 \mathrm{D}$ models, as well as the quality of the critical metadata for use cases such as body landmarks and measurements. To further this goal, The IEEE 3D Group has completed a Comparative Analysis of anthropometric methods.

The aim of this study is twofold: to determine and compare the compatibility and repeatability of different body measurement extraction methods (e.g. body scanners, smartphone apps and traditional anthropometry) using consistent criteria, and to conduct a data collection and corresponding analyses as a first example of the application of the proposed methodology.

This methodology synthesizes different mathematical tools widely used, including statistical models, minimum sample size required for the analyses, several estimators and plots appropriate to describe the data and quantify the repeatability and compatibility of the different measurement extraction methods. It provides commonly used criteria to assess most of the statistics. In addition, it includes a procedure to obtain comparable snapshots of the 3D outcomes from digital technologies that facilitate its assessment by potential data consumers.

The study consisted of two phases: Phase 1 - Portland, Oregon (USA), December 2018 and Phase 2 - Valencia (Spain), October 2019. A total of 133 subjects (61 in Phase 1 and 72 in Phase 2) participated in the study. All participants were selected to cover a wide range of body height and weight. Each subject had 11 body measurements collected twice by different measuring stations (3D scanning booth, smartphone apps and traditional anthropometry experts). Phase 1 included two 3D scanners - Vitus bodyscan (Human Solutions GmbH) and SS20 (Size Stream LLC); and four experts. Phase 2 included two 3D scanners - MOVE4D (Instituto de Biomecánica) and Portal MX (Texel LLC); four smartphone apps -3D avatar body (Instituto de Biomecánica), SS@Home (Size Stream LLC), 3DLOOK (3DLOOK Inc.) and eM+ (PolyU); and two experts (Figure 1). 
An example of the results is presented within figures 2, 3 and 4 . Further details can be found within the white paper of this study. See IEEE 3DBPwebsite for a link to the paper.

https://standards.ieee.org/industry-connections/3d/bodyprocessing.html
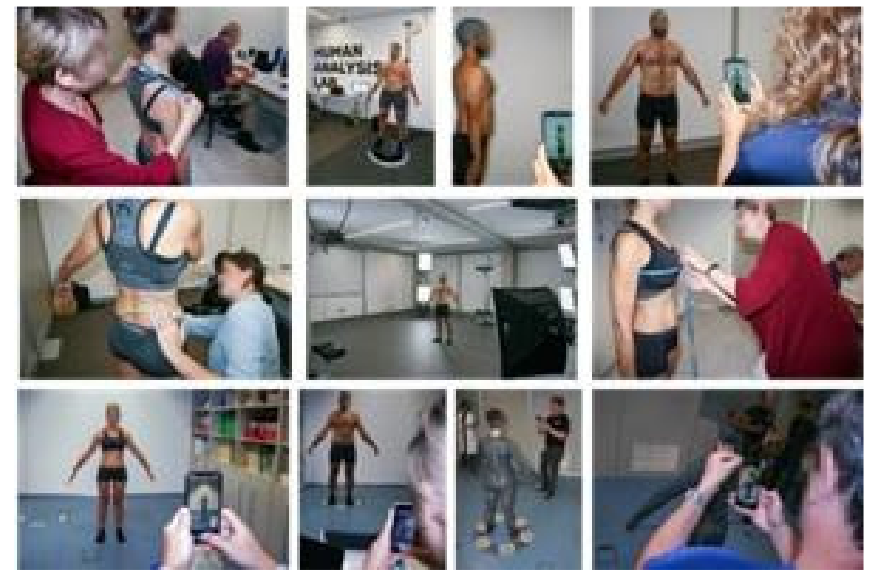

Figure 1: Measuring stations involved in Phase 2.

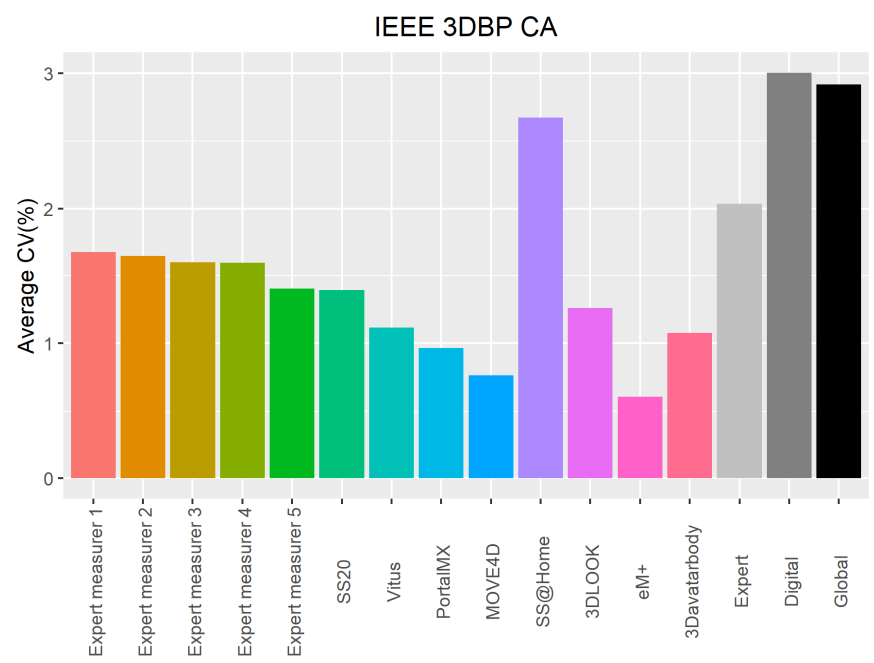

Figure 2: A Repeatability plot, using Global Coefficient of Variation (CV) for each measuring station.

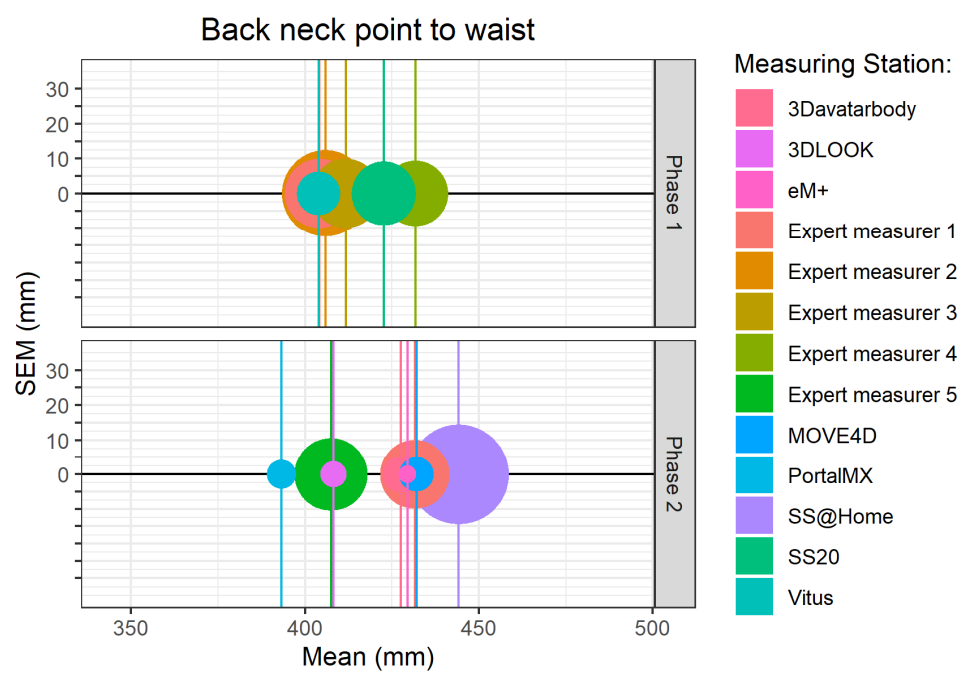

Figure 3: A Compatibility Plot, showing Bias and Standard Error of Measurement (SEM) for "Back neck point-to-waist" measurements. 
Proceedings of 3DBODY.TECH 2020

11th Int. Conference and Exhibition on 3D Body Scanning and Processing Technologies, 17-18 Nov. 2020, Online/Virtual

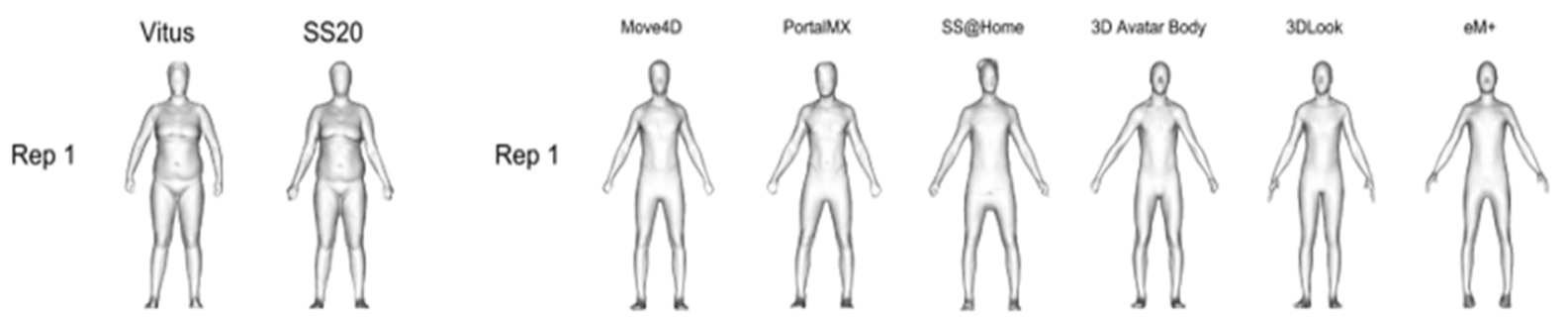

Rep 2

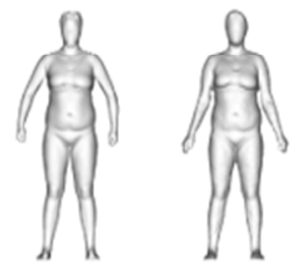

Rep 1
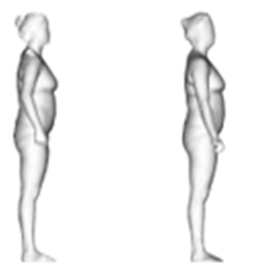

Rep 2

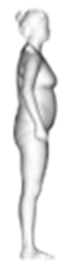

Rep 2
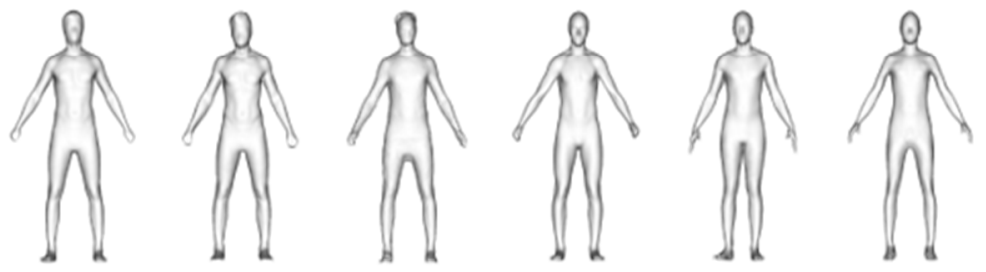

Rep 1
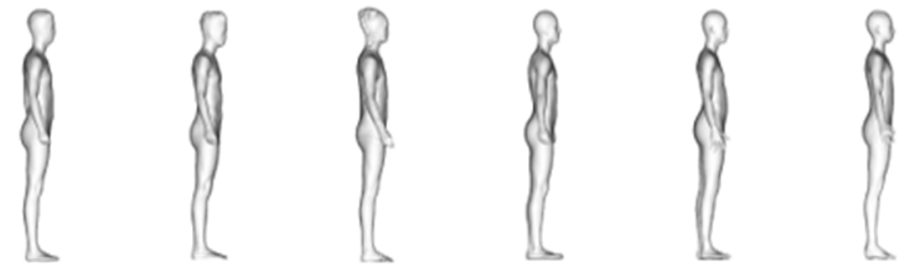

Rep 2
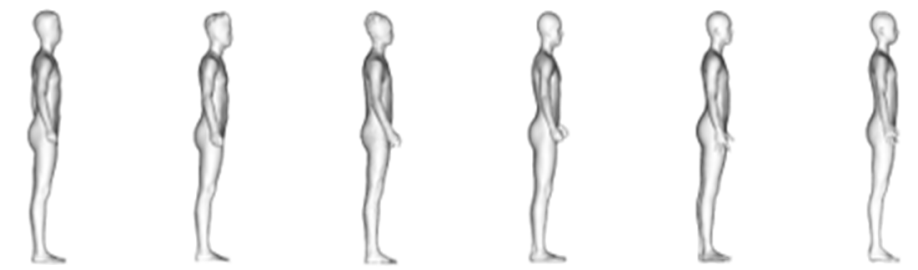

Figure 4: A qualitative assessment of 3D outcomes generated by different digital technologies from Phase 1 (left) and from Phase 2 (right). 